\title{
Acute liver failure secondary to toxic exposure in children
}

\author{
Alina Grama ${ }^{1,2}$, Cornel Aldea ${ }^{3}$, Lucia Burac ${ }^{1}$, Dan Delean³ ${ }^{3}$ Dora Boghitoiu², Bogdan Bulata ${ }^{3}$, \\ Violeta Nitescu², Coriolan Ulmeanu ${ }^{2}$, Tudor Lucian Pop ${ }^{1}$
}

\author{
${ }^{1} 2^{\text {nd }}$ Paediatric Clinic, University of Medicine and Pharmacy Iuliu Hatieganu, \\ Cluj-Napoca, Romania \\ ${ }^{2}$ University of Medicine and Pharmacy Carol Davila, Bucharest, Romania \\ ${ }^{3}$ Nephrology Clinic, Emergency Clinic Hospital for Children, Cluj-Napoca, Romania
}

Submitted: 27 October 2018; Accepted: 3 April 2019

Online publication: 9 September 2019

Arch Med Sci 2022; 18 (1): 84-91

DOI: https://doi.org/10.5114/aoms.2019.87716

Copyright (c) 2019 Termedia \& Banach

\begin{abstract}
Introduction: Acute liver failure (ALF) is a syndrome defined by jaundice, coagulopathy (INR > 1.5) and hepatic encephalopathy in patients with no evidence of prior liver disease. Toxins and drugs are a frequent cause of ALF in children. Material and methods: The aim of our study was to establish the causes of toxic ALF in children followed up in our hospital in the period of January 2000 to August 2018. We retrospectively studied all hospital records of patients who developed ALF after mushroom/drug exposure and had been admitted to our hospital, the main pediatric toxicology center in north-western Romania. Results: In the last 18 years, 123 patients were admitted to our clinic with toxic ALF (89 patients secondary to mushroom ingestion and 34 patients after drug exposure). In the 2000-2012 period accidental mushroom poisoning was the leading cause of toxic ALF. Unfortunately, during the last years, voluntary drug ingestions have increased dramatically. The most commonly incriminated drug was acetaminophen (52.94\%).

Conclusions: ALF in mushroom poisoning is associated with a high mortality in children, despite optimal medical therapy. This etiology was one of the most important causes of death in our cohort. The difficulty in accessing emergency liver transplantation is an obstacle common to many Eastern European pediatric centers. Fortunately, in the last 5 years the incidence of mushroom intoxications has decreased in our area. It is worrying that over the last few years there has been an increased incidence of toxic ALF after drug exposure (for suicidal purposes or due to lenient regulations for prescribing hepatotoxic medications).
\end{abstract}

Key words: children, drugs, liver, mushrooms, acute liver failure, toxic.

\section{Introduction}

Acute liver failure (ALF) in children is a rare disease with a high mortality rate in the absence of emergency liver transplantation. ALF is responsible for $10-15 \%$ of all pediatric liver transplantations [1]. A consensus reached by the PALF (Pediatric Acute Liver Failure) study group defined the criteria for ALF in children as no evidence of a preexisting chronic liver disease, hepatic-based coagulopathy that is not corrected by parenteral administration of vitamin $\mathrm{K}$, international normalized ratio (INR) between 1.5 and 1.99 in the presence of encephalopathy, or INR greater than 2.0 in the absence of encephalopathy [2, 3]. The etiology of ALF in children and

\author{
Corresponding author: \\ Alina Grama MD \\ $2^{\text {nd }}$ Paediatric Clinic \\ University of Medicine \\ and Pharmacy luliu \\ Hatieganu Cluj-Napoca \\ 3-5 Crisan Street \\ 400177 Cluj-Napoca, \\ Romania \\ Phone: 0040747170534 \\ E-mail: \\ gramaalina16@yahoo.com
}


adults is different and varies with age. In neonates and infants, infections and metabolic disorders are more frequent, while in adolescents ALF occurs mainly in toxic hepatitis and Wilson disease $[2,3]$.

Toxic-induced liver injury is the second most common cause of ALF in children, after viral causes, with a prevalence between $20 \%$ and $25 \%$ [4]. The most important causes involved are mushroom poisoning and drugs (acetaminophen, valproic acid, albendazole, carbamazepine) [5].

Amanita phalloides is responsible for almost $90 \%$ of the deaths occurring after mushroom ingestion. The toxicity of $A$. phalloides is related to two distinct groups of toxins: phallotoxins and amatoxins. Phallotoxins are not absorbed from by intestine and do not cause hepatotoxicity $[1,6]$. These are responsible for the damage of the enterocytes, thus producing gastrointestinal symptoms (nausea, vomiting, diarrhea, abdominal pain). The amatoxins are responsible for the liver destruction and are associated with ALF in children $[1,6]$. The toxins are not destroyed by any form of cooking and the lethal dose is very low: in adults $0.1 \mathrm{mg} / \mathrm{kg}$ can cause death [6]. The toxic dose is lower for a child and liver damage can differ in severity from the mature liver, similar to other causes such as ischemia/reperfusion injuries [7]. Amatoxins are quickly absorbed from the gastrointestinal tract, enter the circulation and are transported by a nonspecific transport system to the liver. In hepatocytes, the toxins interfere with RNA-polymerase II, impair transcription of DNA to RNA and cause fatty degeneration, lipid and carbohydrate accumulation in the cell nuclei, and extensive centrilobular necrosis and hemorrhage $[6,8]$. A. phalloides toxins also affect other organs: kidneys, where the toxins produce acute tubular necrosis, pancreas, testicles or adrenal glands [6].

Drugs can cause severe hepatic injury through various mechanisms: a dose-dependent response (acetaminophen, halothane), an idiosyncratic reaction (albendazole, isoniazid, antibiotics, NSAIDs) or a synergistic reaction (amoxicillin + clavulanic acid, trimethoprim sulfamethoxazole) [9]. Hepatotoxicity is due to hepatocyte damage or canalicular membrane disruption. The activation of the T-cell system or tumor necrosis factor- $\alpha$ causes hepatocyte apoptosis or inhibition of mitochondrial function, leading to decreased production of ATP [10].

The aim of our study was to analyze the main causes of toxic ALF in children hospitalized in our pediatric clinic with a specialized department of toxicology. Our hospital admits patients mostly from the North-Western part of Romania.

\section{Material and methods}

We retrospectively studied the medical files of all patients with ALF after toxic substance in- gestion who were hospitalized between January 2000 and August 2018. During this period, 1143 children were admitted for toxic substance ingestion: 326 patients with mushroom poisoning (89 developed ALF) and 917 patients with accidental or voluntary ingestion of different drugs (75 patients developed hepatotoxicity, with 34 cases complicated with ALF).

The inclusion criteria were: age under 18 years, toxic ingestion reported by our patient or by their relatives and diagnosis of ALF, based on the PALF criteria (INR > 1.5 in the presence of clinical encephalopathy or INR > 2 in the absence of encephalopathy in patients without a previous chronic liver disease). Other possible etiologies of ALF were excluded (metabolic disorders, viral hepatitis A, B, C, CMV, EBV, herpesvirus, Wilson's disease and autoimmune hepatitis).

In the cases of patients with mushroom poisoning, we analyzed data related to the age and sex distribution, the type of mushroom preparation (the amount of toxin ingested is greater in the case of roasting), the socio-economic level and living conditions of these patients, the weather conditions of the time period, the seasonal variations and the influence of the legislative changes and media campaign in reducing the incidence of these cases.

For patients with drug ingestion we analyzed the age and sex distribution, the type of ingestion (voluntary or accidental), the ingested dose (therapeutic or overdose), the type of administration (enteral or intravenous) and the outcome of these cases.

We excluded patients without criteria for ALF or those with other possible causes of ALF (a 7-month old baby admitted to our hospital with ALF after paracetamol overdose but who was later proven to have hereditary fructose intolerance, 2 patients with mushroom ingestion who had positive immunology for hepatitis $A$, and 2 patients with mononucleosis who received overdoses of acetaminophen). We also excluded oncology patients who developed ALF after chemotherapy (considering the increased incidence or hepatotoxicity among these patients). Also, children with hepatitis A were not part of our study because they are hospitalized in the Infectious Disease Hospital. Six other children were admitted with ALF and clinical suspicion of Reye syndrome. We did not include them because of their fast clinical deterioration and the lack of time to carry out the necessary laboratory investigations.

Most cases of acetaminophen overdose or mushroom poisoning were treated by stomach irrigation, glucose and electrolyte infusions, diuretics or activated charcoal. All of them received 
Table I. Characteristics of the patients with ALF

\begin{tabular}{|lccc|}
\hline Patient demographics & $\begin{array}{c}\text { Mushroom poisoning ALF - } \\
\text { 89 patients }\end{array}$ & $\begin{array}{c}\text { Drug ingestion ALF - } \\
\text { 34 patients }\end{array}$ & $P$-value \\
\hline Age (mean \pm SD) [years] & $7.78 \pm 3.96$ & $12.26 \pm 4.52$ & $<0.00001$ \\
\hline Gender: girls, $n(\%)$ & $39(48.83)$ & $26(76.47)$ & 0.0012 \\
\hline Encephalopathy, $n(\%)$ & $63(70.78)$ & $5(14.70)$ & $<0.00001$ \\
\hline Jaundice, $n(\%)$ & $50(56.17)$ & $11(32.35)$ & $<0.00001$ \\
\hline Acute renal failure, $n(\%)$ & $14(14.28)$ & $1(2.94)$ & 0.0369 \\
\hline AST (mean \pm SD) $[$ IU/l] & $2959.02 \pm 4439.40$ & $654.44 \pm 853.73$ & 0.0033 \\
\hline ALT (mean \pm SD) $[$ IU/l] & $2629.43 \pm 3301.16$ & $681.53 \pm 907.71$ & 0.0009 \\
\hline Bilirubin (mean) $[\mathrm{mg} /$ dl] & $2.67 \pm 2.49$ & $3.31 \pm 4.31$ & 0.3124 \\
\hline INR (mean) & $3.91 \pm 4.06$ & $2.23 \pm 1.12$ & 0.0221 \\
\hline $\begin{array}{l}\text { Extracorporeal blood purification } \\
\text { methods, } n(\%)\end{array}$ & $62(69.66)$ & $2(5.88)$ & $<0.00001$ \\
\hline Survival, $n$ (\%) & $43(48.31)$ & $32(94.11)$ & $<0.00001$ \\
\hline
\end{tabular}

ALF-acute liver failure.

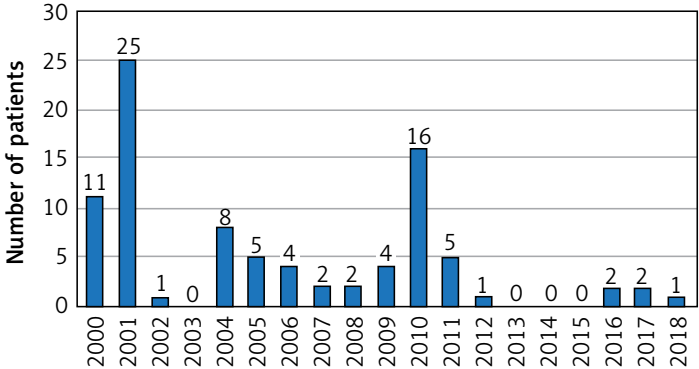

Figure 1. Yearly distribution of patients with acute liver failure after mushroom poisoning

$\mathrm{N}$-acetylcysteine and all had a complete recovery. In mushroom poisoning, $81.93 \%$ of patients received high doses of penicillin G $(1,000,000 \mathrm{lU} /$ $\mathrm{kg} /$ day) and 67 patients (75.28\%) were treated with hemoperfusion, plasma exchange or hepatic dialysis (molecular adsorbent recirculating system (MARS) or fractioned plasma separation and adsorption (FPSA)). The most common method was hemoperfusion on activated charcoal cartridge (47 patients). Plasma exchange was used in 11 patients ( 5 of them survived) and liver dialysis (MARS/FPSA) was performed in 4 patients (all of them survived). Extracorporeal treatment methods have been used less for drug toxicities: in 1 case of valproic acid overdose and for a child with isoniazid toxicity.

In all cases of bleeding or coagulopathy, fresh frozen plasma and vitamin $\mathrm{K}$ were administered. Lactulose, mannitol and arginine infusion have been used to treat hepatic encephalopathy.

In the case of albendazole or isoniazid toxicity, corticosteroid therapy has been used due to the associated autoimmunity. Short corticosteroid courses $(1 \mathrm{mg} / \mathrm{kg} /$ day) were used in 8 cases with quick remission in 2-3 months.

\section{Results}

During the period of January 2000 to August 2018, 123 children with toxic ALF were hospitalized: 89 after accidental ingestion of wild mushrooms and 34 after drug ingestion. Among those who consumed mushrooms the mean age was $7.84 \pm 3.85$ while the mean age in drug ingestion patients was $12.29 \pm 4.34(p<0.00001)$.

\section{Mushroom poisoning}

Of all victims of mushroom poisoning, 39 (48.83\%) were girls and 50 (56.17\%) were boys. The gender distribution changes significantly in those who received/took drugs: higher incidence in girls $(76.47 \%)$ compared with boys $(23.52 \%)$ ( $p=$ $0.0057)$. Table I presents the characteristics of the patients with ALF included in this study.

ALF after mushroom poisoning was more frequent in 2001 ( 25 cases), 2010 (16 cases) and 2000 (11 cases), representing the leading cause of death after toxic exposure in our hospital (46 cases) (Figure 1).

Mushroom poisoning occurs more frequently during the summer and autumn, because heavy rainfall specific to these seasons favors mushroom growth and encourages forest foraging in local rural communities (Figure 2).

The methods of cooking the ingested mushrooms were roasting in 35 (30.08\%) cases and stewing in $54(60.67 \%)$ cases.

The most common first-noticed symptoms in mushroom poisoning patients were gastrointestinal (vomiting and/or diarrhea), but other organs were also affected. Encephalopathy was present in $63(70.78 \%)$ children and acute kidney injury (oliguria with increased serum creatinine level) in 14 (14.28\%) patients. Death occurred in 46 (51.68\%) children after mushroom poisoning. 


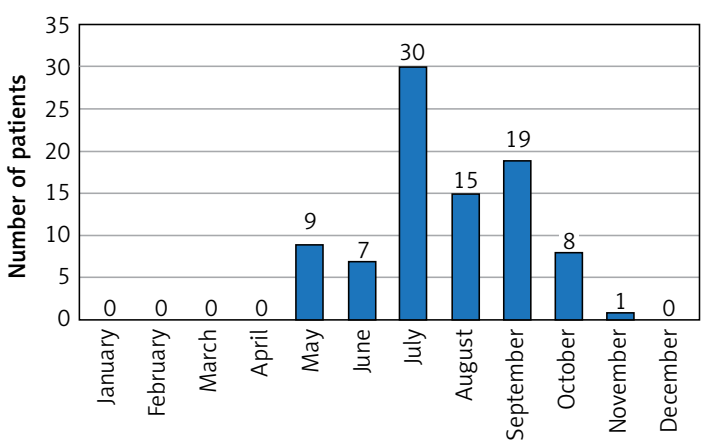

Figure 2. Acute liver failure after mushroom poisoning

\section{Drug ingestion}

During the last 6 years, the number of drug-induced ALF has increased dramatically: 34 cases since 2012 (Figure 3). Of these, 18 (52.94\%) patients were hospitalized after acetaminophen overdose for suicidal purposes, 11 (32.35\%) patients developed ALF after they were treated with albendazole for intestinal parasitosis, 2 patients developed ALF after isoniazid therapy and 1 patient each with ALF after fluconazole, colchicine or valproic acid.

Table II presents the most important clinical manifestations in ALF patients after mushroom and drug poisonings.

Risk factors for fatal evolution in acute liver failure patients

The overall mortality rate for toxic ALF in our study was high (39.02\%), mostly from mushroom poisoning. There were two deaths after drug ingestion (5.88\% out of all drug-induced ALF patients admitted). In our cohort, the risk factors for death in ALF were: encephalopathy (present in $47 / 48$ patients with fatal outcome, $97.92 \%$ vs. $11 / 75$ alive patients, $14.67 \%, p<0.00001)$, presentation level of serum ALAT $(3331.87 \pm 4016.18 \mathrm{UI} / \mathrm{dl}$ in deceased patients vs. $1296.81 \pm 1644.90 \mathrm{UI} / \mathrm{dl}$ in alive patients, $p=0.0001)$, ASAT (4066.90 $\pm 5634.14 \mathrm{Ul} / \mathrm{dl}$ in deceased patients vs. 1205.24 $\pm 1458.50 \mathrm{Ul} / \mathrm{dl}$ in alive patients, $p<0.0001$ ), total bilirubin $(3.67 \pm 2.95 \mathrm{mg} / \mathrm{dl}$ in deceased patients

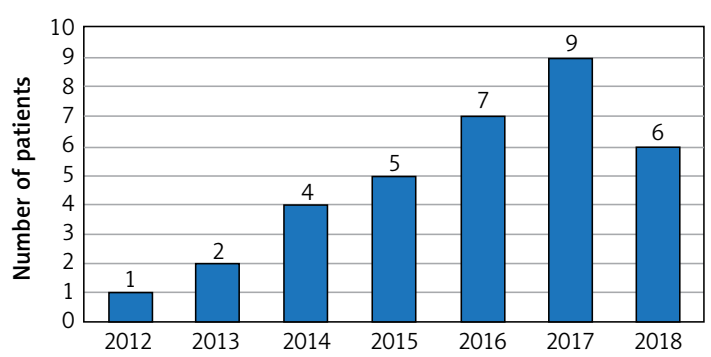

Figure 3. Yearly distribution of patients with acute liver failure after drug ingestion vs. $2.32 \pm 3.09 \mathrm{mg} / \mathrm{dl}$ in alive patients, $p=0.0181$ ), INR $(6.01 \pm 4.31$ in deceased patients vs. $3.01 \pm 3.38$ in alive patients, $p<0.00001)$ and acute kidney injury (present in 15/48 patients with fatal outcome, $31.25 \%$ vs. $1 / 75$ alive patients, $1.33 \%, p<0.00001$ ).

\section{Discussions}

Based on the latest official data reported by the World Health Organization, poisoning represents the third cause of unintentional injury death in Europe [11]. Although rare in the pediatric population, ALF retains a high mortality rate in the absence of emergency liver transplantation [1-3]. Unfortunately, our country does not have a national registry for ALF. For this reason, epidemiological data are incomplete, making a cross-European comparison of drug-induced ALF very difficult.

Mushroom poisoning is an important cause of toxic ALF in our country and is a worldwide problem. The earliest literature description of a fatal mushroom poisoning was made by the Greek poet Euripides. He described in a manuscript the death of his wife and three children after eating poisonous mushrooms $[6,7]$. It is estimated that there are over 5000 species of mushrooms worldwide, especially in forested mountainous areas. of these, Amanita phalloides is responsible for most deaths occurring after mushroom ingestion - hence its common name of death cap. In Western Europe studies report approximately 50-100 fatal cases per year $[6,7]$.

Table II. Clinical manifestations in patients with acute liver failure due to mushroom and drugs poisoning

\begin{tabular}{|lcccc|}
\hline Clinical manifestations & Mushrooms poisoning & Acetaminophen & Albendazole & Other drugs \\
\hline Jaundice & ++++ & $+/-$ & ++ & - \\
\hline Encephalopathy & +++ & $+/-$ & ++ & + \\
\hline Coagulopathy & ++++ & + & + & ++ \\
\hline Hypoglycaemia & +++ & - & + & + + \\
\hline Hepatomegaly & ++ & - & + \\
\hline Ascites & + & +++ & + & + \\
\hline Gastrointestinal manifestations & +++ & + & + \\
\hline
\end{tabular}

$+/$ - present or absent, + up to $25 \%$ of cases, $++25-50 \%$ of cases, $+++50-90 \%$ of cases, ++++ more than $90 \%$ of cases. 
Table III. Annual distribution of rainfall in Romania [11]

\begin{tabular}{|lcc|}
\hline \multirow{2}{*}{ Decade } & \multicolumn{2}{c|}{$21^{\text {st }}$ century } \\
\cline { 2 - 3 } & Dry years & Rainy years \\
\hline $2001-2010$ & $2000,2002,2006,2007,2008,2009$ & $2001,2004,2005,2006,2008,2010$ \\
\hline $2011-2020$ & $2011,2012,2013$ & - \\
\hline
\end{tabular}

Mushroom poisoning was the most common cause of ALF in children from north-western Romania. In the period January 2000 to August 2018, 329 children with mushroom poisoning were hospitalized in our clinic and 89 (27.05\%) developed ALF, with a high mortality: 46 (51.68\%) cases. ALF after mushroom ingestion was more frequent in 2001 (25 cases), 2010 (16 cases) and 2004 (8 cases). According to data reported by the Romanian National Agency of Meteorology, 2001, 2004, 2006, 2008 and 2010 were the rainiest years of this period (Table III). This elicited increased foraging and mushroom consumption, as well as a high number of children developing severe hepatotoxicity. At the opposite end of the rainfall spectrum, in 2011-2012 (described as some of the driest years), the number of cases of ALF after mushroom poisoning decreased significantly. An overall increase in rural living standards and better public information campaigns were two other factors that contributed to this drop in wild mushroom consumption.

Mushroom poisoning occurs more frequently during the summer and autumn because seasonal rains are more frequent in these seasons and mushroom foraging is a popular hobby in Romanian villages during these periods. This is also one of the times of the year when there are fasting periods in the Orthodox Christian tradition. In these days/weeks of fasting, according to Orthodox rules, many people do not eat meat, eggs or dairy products, the daily menu being based on vegetables, fruits, mushrooms and soy.

The mushroom cooking methods were also important. In most cases the mushrooms were cooked in a stew, thus dispersing the toxins. If the mushrooms were roasted, in most of the cases only one family member was affected.

Fortunately, since 2012 the frequency of ALF due to mushrooms has decreased and, subsequently, mortality has also declined. This is also confirmed by the reports of the Public Health Department. It has periodically issued warnings to the population about the devastating effects of mushroom poisoning and launched an important campaign to educate the population through mass media. At the same time, the legislative framework was amended to restrict foraging and trade rights for land owners and members of rural communities. The Environmental Protection Agency established higher penalties and closer moni- toring of illegal trade $[12,13]$, resulting in a drop in severe intoxications.

Even though the mortality rate of all mushroom poisoning cases (329 cases) was low (13.98\%), in the ALF subgroup the mortality was high (51.68\%) despite the intensive care treatment (including hemoperfusion, plasmapheresis and hepatic dialysis MARS/FPSA). The high mortality rate was also due to late presentation to the hospital and to the impossibility to perform emergency liver transplant in our patients.

The risk factors for death in these patients were encephalopathy, level of transaminases and total bilirubin, severity of coagulopathy and presence of acute kidney injury. In a small cohort of 17 children with ALF due to mushroom poisoning (of whom 9 died), PELD score over 20 at admittance predicted very accurately the mortality of the patients, better than King's College Criteria. The PELD score was significantly increased in deceased patients $(45.9 \pm 11.8$ vs $-3.6 \pm 7.0, p=0.0000001)$. In these patients, emergency liver transplantation is indicated, and the patients should be referred to specialized liver units [14].

Another analysis of mushroom patients showed that high serum creatinine level and the presence of encephalopathy could predict mortality in children with ALF [15]. When analyzing the factors responsible for the difference between survival and fatal outcome in children with ALF, the most important were age, serum potassium level, and total bilirubin level (measured on the $3^{\text {rd }}$ day). Transaminase, hemoglobin, thrombocyte, and albumin levels showed little prognostic benefit [16]. Peng et al. stated that Child-Pugh, model for end-stage liver disease (MELD), and albumin-bilirubin (ALBI) scores might be ineffective in predicting the mortality in acute-on-chronic liver failure (ACLF) adult patients with cirrhosis [17].

The epidemiological data remain ambiguous in many countries, especially in EU non-member states. Around 50-100 fatal cases of mushroom poisoning are described every year in Europe [6, 18, 19]. In Turkey, in 2010, 294 patients (204 adults and 90 children) were admitted to the Pediatric and Adult Emergency Internal Medicine and ICU departments in the Cumhuriyet University Hospital between 2000 and 2007 with mushroom poisoning. The mortality rate was very low $(0.1 \%)$, with only three recorded deaths [20]. Another Portuguese study gathered data from 10 regional cen- 
ters between 1999 and 2000. They found 93 patients with mushroom poisoning (32 children), with a $6.8 \%$ mortality rate [21]. In Poland, as in our country, mushroom picking is common, especially in rural areas [22]. In Northeast Iran, during 20052011, 32 children were admitted with mushroom poisoning, with a mortality rate of $22 \%[23,24]$.

Drugs are an important cause of liver injury, representing $10-15 \%$ of patients hospitalized with ALF [25]. During the last years, the number of drug-induced ALF has increased dramatically in our country. There were 34 cases of ALF due to drug exposure, with an incremental increase every year. The mortality rate after drug exposure was $5.88 \%$. Unfortunately, most of these cases are selfharm situations and occurred among adolescents.

According to data published by Eurostat in 2015, Romania ranks $13^{\text {th }}$ in Europe in the number of suicides in the adolescent population, with an average of 5 cases/100,000 people [26]. Family issues, parental divorce, loss of a loved one, and school envirenment have been proven to be the main causes of suicide attempts in our patients. It is our belief that the main goal of the drug ingestion was to draw attention to one's problems, without realizing the risk. Acetaminophen was one of the most used drugs for this purpose.

In Romania, acetaminophen is an over-thecounter (OTC) drug, which makes it easily available and an attractive option for teenage self-harm. Fortunately, the use of acetylcysteine as an antidote in acetaminophen intoxication has caused the mortality among our cases to remain low (no case in our study).

In the whole world, acetaminophen is the most common cause of drug-induced acute hepatitis and an important cause of ALF. Generally, is a dose-dependent hepatotoxic agent, and it is safe in therapeutic doses [3]. The hepatotoxic dose is over $150 \mathrm{mg} / \mathrm{kg}$ for children under the age of 6 and over $200 \mathrm{mg} / \mathrm{kg}$ for those older than 6 years. Overdose of acetaminophen produces direct hepatotoxicity and hepatocellular necrosis. Hepatic injury generally starts 24 to $72 \mathrm{~h}$ after ingestion. The clinical symptoms usually appear later (48-96 h after ingestion): jaundice, confusion, hepatic failure and, in some cases, death [10]. In our study all acetaminophen ingestions were intentional. There were 18 patients with ALF after acetaminophen overdose, aged between 12 and 18 years old. The ingested doses were between $160 \mathrm{mg} / \mathrm{kg}$ and $600 \mathrm{mg} / \mathrm{kg} /$ dose and all these patients survived due to early presentation at the hospital, shortly after ingestion (within 1-24 h) and quick administration of $\mathrm{N}$-acetylcysteine.

The second cause of ALF due to drugs in our patients was albendazole. This is commonly prescribed in our country for the treatment of many types of intestinal parasitosis (proven or suspected). It is our opinion that over-prescription leads to an increased number of patients hospitalized with toxic hepatitis. Generally, albendazole toxicity is immune mediated. Liver injury usually manifests with increased transaminase levels, with mild/ moderate forms of acute hepatitis, but in some patients it may progress to ALF [27]. Immune-mediated injury as well as idiosyncratic reaction (polymorphism of CYO3A4 enzyme) can induce periportal invasion of inflammatory cells, periportal necrosis and periportal steatosis. In our hospital, 14 children have been admitted during the last 5 years with acute hepatitis after albendazole ingestion and 11 of them developed ALF. Five patients had increased total IgG levels and positive antinuclear antibodies (ANA). They all had rapidly favorable outcomes (5-8 weeks) with hepatoprotective therapy (silymarin) or ursodeoxycholic acid (UDCA). Some patients required prednisone. There is no antidote for albendazole hepatotoxicity [28]. Glucocorticoids could be used in the patients with toxic acute liver failure, especially if they are associated with autoimmunity elements [29]. Use of albendazole only in documented cases of parasitosis such as hydatid cyst, aspergillosis or other parasitosis with severe manifestations would reduce the incidence of these cases with severe hepatotoxicity [30]. There are limited data about liver toxicity secondary to antiparasitic therapy in children. Independent drug safety studies such as a recently published article about the safety of 15 drugs used in patients with rheumatoid arthritis would be helpful to clinicians [31].

Isoniazid (INH) caused severe hepatotoxicity in two of our patients. The first case was an accidental overdose, which caused an acute reaction immediately after the exposure. The second was a chronic intoxication, occurring a few weeks after the initiation of tuberculostatic therapy. In this case autoimmune features coexisted, and we presumed an idiosyncratic mechanism for the hepatotoxicity [31].

Valproic acid (valproate) is an organic acid frequently used in the treatment of epilepsy, isolated seizures and other neurological and psychiatric child disorders [32]. It can produce hyperammonemia with minimally increased or normal transaminases, acute cholestatic hepatitis, ALF and/or Reye-like syndrome. The mechanism of valproic acid toxicity is mitochondrial damage with loss of the function. The antidote is oral or intravenous carnitine $[33,34]$. We had 4 patients with hepatotoxicity secondary to valproate overdose and 1 patient who developed severe hepatotoxicity with ALF. The latter was a girl, 6 years old, with post-traumatic epilepsy, who was accidentally overdosed with valproic acid. 
Colchicine is an alkaloid plant used for treatment of gout. Usually colchicine is not hepatotoxic except when it is used in large doses [35]. A 16-year-old boy developed ALF and died after ingestion of an overdose of colchicine $(60 \mathrm{mg})$ with suicidal intent.

Fluconazole is a fungicidal agent frequently used orally or intravenously for the treatment of systemic or localized fungal infections (Candida, Cryptococcus). About 5\% of the patients treated with fluconazole developed mild hepatotoxic phenomena and, rarely, ALF [36, 37]. A 2-year-old boy with chronic granulomatous disease developed ALF after fluconazole therapy administered for severe and repeated fungal systemic infections. He died later due to primary disease complications.

In conclusion, toxic ALF is associated with a high mortality in children, despite optimal medical therapy including hemoperfusion, plasmapheresis or hepatic dialysis (MARS/FPSA). The optimal therapeutic solution is emergency liver transplantation, which was not readily available for patients in our center. In the first decade of our study period, mushroom poisoning was the most common cause of pediatric ALF in North-Western Romania and it was associated with a high mortality rate. During the last 7 years, the number of ALF secondary to mushroom ingestion has decreased significantly. Improvement of health education and standard of living in our country has played an important role in reducing the frequency of mushroom poisoning. Unfortunately, in the last 5 years we have seen a rise in self-harm through drug overdose causing ALF. The most commonly used was acetaminophen due to easy access (OTC drug). Many commonly used drugs are overprescribed, resulting in hepatic toxicity and, in severe cases, ALF or death.

\section{Acknowledgments}

Alina Grama and Cornel Aldea contributed equally to this work.

\section{Conflict of interest}

The authors declare no conflict of interest.

\section{References}

1. Colleti J Jr, Azevedo RT, de Calvalho WB. Pediatric acute liver failure: current perspectives. Liver Res Open J 2017; 2: $14-5$.

2. Squires RH. Acute liver failure in children. Semin Liver Dis 2008; 28: 153-66.

3. Dhawan A. Etiology and prognosis of acute liver failure in children. Liver Transpl 2008; 14: S80-4.

4. Ichai P, Didier S. Epidemiology of liver failure. Clin Res Hepatol Gastroenterol 2011; 35: 610-7.

5. Squires RH, Shneider LB, Bucuvalas J, et al. Acute liver failure in children: the first 348 patients in the pediat- ric acute liver failure study group. J Pediatr 2006; 148: 652-8.

6. Santi L, Maggioli C, Mastroroberto M, Tufoni M, Napoli L, Caraceni P. Acute liver failure caused by amanita phalloides poisoning. Int J Hepatol 2012; 2012: 487480.

7. Trocha M, Merwid-Ląd A, Pieśniewska M, et al. Agerelated differences in function and structure of rat livers subjected to ischemia/reperfusion. Arch Med Sci 2018; 14: 388-95.

8. Mengs U, Pohl RT, Mitchell T. Legalon ${ }^{\oplus}$ SIL: the antidote of choice in patients with acute hepatotoxicity from amatoxin poisoning. Curr Pharm Biotechnol 2012; 13: 1964-70.

9. Dhawan A. Acute liver failure in children and adolescents. Clin Res Hepatol Gastroenterol 2012; 36: 278-83.

10. Mehta N, Ozick LA, Gbadehan E. Drug-induced hepatotoxicity. Medscape. http://emedicine.medscape.com. Accessed: March 20, 2018.

11. Sandu I. Climate changes in Romania and impacts on water resources in agriculture. Bucharest 2013. http:// www.meteoromania.ro. Accessed: September 27, 2018.

12. Order no. $410 / 2008$ about the approval of the authorizing procedure about catching, acquiring and/or marketing, on the national or export territory, of moss flowers, plant fossils and fossils of vertebrate and invertebrate animals, as well as of plants and animals from flora and wildlife, respectively, and their import. The Romanian Official Monitor, August 2010.

13. Law no. $171 / 2010$ regarding the establishment and sanctioning of forest contraventions. Romanian Official Monitor, May 2008.

14. Pop TL, Grama A, Stefanescu A, Delean D, Aldea C, Bizo A. PELD score as a prognostic factor in fulminant liver failure caused by mushroom poisoning in children. J Hepatol 2016; 64 (Suppl 2): S304-5.

15. Stefanescu A, Pop TL, Stefanescu H, Feier D, Bizo A, Miu N. Serum creatinin and the presence of encephalopathy at presentation may predict mortality in children with acute liver failure. J Hepatol 2013; 58 (Suppl 1): s417.

16. Pop HF, Sarbu C, Stefanescu A, Bizo A, Pop TL. Prognostic factors in liver failure in children by discriminant analysis of clinical data. A chemometric approach. Studia UBB Chemia 2015; 40: 101-8.

17. Peng Y, Qi X, Tang S, et al. Child-Pugh, MELD, and ALBI scores for predicting the in-hospital mortality in cirrhotic patients with acute-on-chronic liver failure. Expert Rev Gastroenterol Hepatol 2016; 10: 971-80

18. Sethi D, Towner E, Vincenten J, Gomez MS, Racioppi F. Poisoning in European report. In Sethi D, Towner E, Vincenten J, et al. (eds.). Child European Prevention. WHO Europe 2008; 39-45.

19. Eren SH, Urgulu S, Demirel Y, Kukul F. Mushrooms poisoning: retrospective analysis of 294 cases. Clinics 2010; 65: 491-6.

20. Mas A. Mushrooms, amatoxins and the liver. J Hepatol 2005; 45: 166-9.

21. Brandão JL, Pinheiro J, Pinho D, et al. Mushroom poisoning in Portugal. Acta Med Port 2011; 24 (Suppl 2): 269-78.

22. Sein Anand J, Chodorowski Z, Wiśniewski M, Waldman W. The assessment of albumin liver dialysis-MARS efficacy in the treatment of Amanita phalloides poisoning. Przegl Lek 2007; 64: 255-7.

23. Varonschi M, Naghili B. Mushrooms poisoning in Northwest of Iran. Iran J Clin Infect Dis 2007; 2: 169-75.

24. Dadpour B, Tajoddini S, Rajabi M, Afshari R. Mushroom poisoning in the northeast of Iran; a retrospective 6-year epidemiologic study. Emerg (Tehran) 2017; 5: e23. 
25. Farrell SE, Miller MA. Acetaminophen toxicity. http:// emedicine.medcape.com. Accesed: March 2018.

26. Sarbu EA. The lifeline for suicide prevention of children and teenagers in Bucharest, Romania. Sociology Study 2015; 5: 415-27.

27. Freire JF, Rocha LMC, Lima LG, Raposo PRA. Subfulminant acute liver failure by albendazole: case report. J Med Cases 2015; 6: 342-5.

28. Shah C, Mahapatra A, Shukla A, Bhatia S. Recurrent acute hepatitis caused by albendazole - case report. Trop Gastroenterol 2013; 34: 38-9.

29. Bai Z, Li H, Zheng K, et al. A good response to glucocorticoid for sub-acute liver failure: a case report. Transl Gastroenterol Hepatol 2018; 3: 25.

30. Sokouti M, Sadeghi R, Pashazadeh S, et al. A systematic review and meta-analysis on the treatment of liver hydatid cyst using meta-MUMS tool: comparing PAIR and laparoscopic procedures. Arch Med Sci 2019; 15: 284-308.

31. Ma K, Li L, Liu C, Zhou L, Zhou X. Efficacy and safety of various anti-rheumatic treatments for patients with rheumatoid arthritis: a network meta-analysis. Arch Med Sci 2019; 15: 33-54.

32. Drug record. Clinical and Research Information of Drug-Induced Liver Injury. https://livertox.nih.gov/Isoniazid.htm. Accessed: May 2018.

33. Drug record. Clinical and Research Information of Drug-Induced Liver Injury. https://livertox.nih.gov/Valproate.htm. Accessed: May 2018.

34. Pirmohamed M, Leeder SJ. Anticonvulsant agents. In: Drug-induced Liver Disease. 3rd ed. Kaplowitz N, DeLeve LD, (eds.). Elsevier, Amsterdam 2013; 423-41.

35. Carr AA. Colchicine toxicity. Arch Intern Med 1965; 115 : 29-33.

36. Moseley RH. Antifungal agents. Antibacterial and antifungal agents. In: Drug-induced Liver Disease. $3^{\text {rd }}$ ed. Kaplowitz N, DeLeve LD (eds.). Elsevier, Amsterdam 2013; 470-81.

37. Drug record. Clinical and Research Information of Drug-Induced Liver Injury. https://livertox.nih.gov/AntifungalAgents.htm. Accessed: May 2018. 